\title{
Post-Reproductive Health. Opportunity window for the Prevention of Co-Morbidities
}

Víctor Manuel Vargas Hernández*, Lorena Patricia Ferrer Arreola, María Antonia Basavilvazo Rodríguez, María Antonia Valdés Vargas Miembros de la mesa directiva de Asociación Mexicana para el Estudio del Climaterio (AMEC)

*Corresponding Author: Víctor Manuel Vargas Hernández, Insurgentes Sur 605-1403, Nápoles, 03810 D.F. México, Received Date: December 28, 2020; Accepted Date: January 27, 2021; Published Date: February 05,2021

Citation: Vargas Hernández VM, Ferrer Arreola LP, Basavilvazo Rodríguez MA, Valdés Vargas MA (2021) Post-Reproductive Health. Opportunity window for the Prevention of Co-Morbidities. J Women Health Care Issues, 4(1); Doi:10.31579/2642-9756/039

Copyright: () 2021 Magdalene Karon, This is an open-access article distributed under the terms of the Creative Commons Attribution License, which permits unrestricted use, distribution, and reproduction in any medium, provided the original author and source are credited.

\begin{abstract}
Post-reproductive health is challenging, and menopausal hormone therapy (THM) is surrounded by many controversies. It has witnessed a rise and fall lasting several years. We understand that comorbidity increases its incidence beyond menopause and we need to prevent them. When THM was used universally, the risk-benefit ratio became unfavorable, its use fell dramatically; although more recent studies have introduced the concept of window of opportunity, where THM has no ill effects and will in fact prevent comorbidities associated with menopause.
\end{abstract}

Keywords; post-reproductive health; menopause; HRT; MHT Window of opportunity

\section{Background}

Millions of women around the world face health challenges beyond menopause, and a significant proportion of them have a longer life expectancy at birth, while the average age of menopause is only 46 years, therefore it is important to maintain positive post-reproductive health, prevent disease, reduce mortality rates, and improve quality of life. Menopausal hormone therapy (MHT) has documented benefits, although it is also associated with some risks such as breast cancer and thromboembolism, worsening the already increased risks related to morbidity, age and pregnancy. Menopause. In the prevention of comorbidities beyond menopause, MHT is a window of opportunity to reduce the associated risks [1-5].

Worldwide, cardiovascular diseases are the main causes of morbidity and mortality; the risk of cardiovascular diseases increases with age in men and women; however, in women due to the cardioprotective effect of estrogen, the risk is delayed until menopause. Osteoporosis-related fractures and cancer are two other major morbidities. Cerebrovascular events, pulmonary embolism, dementia, breast cancer, and endometrial cancer (can occur as a side effect of MHT, The highest incidence of heart disease in women occurs after menopause, estrogen / progesterone MHT, to reduce the risk of heart disease, was prescribed to menopausal women prior to the results of the Health Initiative study of Women (WHI) 6. Table 1;

\begin{tabular}{|l|}
\hline the results of WHI \\
\hline Morbidities related to age, menopause and MHT \\
\hline Cardiovascular morbidities \\
\hline Deep venous and pulmonary embolism \\
\hline Dyslipidemia \\
\hline Diabetes \\
\hline Dementia \\
\hline
\end{tabular}

\begin{tabular}{|l|}
\hline Breast cancer \\
\hline Endometrial cancer \\
\hline Ovarian cancer \\
\hline Cervical cancer \\
\hline
\end{tabular}

Table 1

\section{Osteoporosis-related fractures}

The study of the Women's Health Initiative (WHI) with Heart and Estrogen and Progestin Replacement (HERS) 6 concluded that THM is not recommended for primary or secondary prevention of coronary heart disease; which decreased its universal use after menopause. Although the WHI trial was stopped after 7 years, 27,347 postmenopausal women between the ages of 50 and 79 were followed up for an extended period of 13 years without treatment.

Despite awareness of MHT-related issues, postmenopausal women now seek help only if they have symptoms, some to stay young forever and improve quality of life. Universal use of MHT is no longer recommended, so how do we select women to prescribe MHT, to control symptoms, prevent comorbidities and give them a better quality of life? What strategies should we use to achieve the best balance between the risks and benefits of THM? The answers to these questions are not very clear, but the recently introduced "window of opportunity" concept can be helpful in achieving the objectives.

The concept of "time hypothesis" or "window of opportunity" for the initiation of the THM; indicates that MHT is best given immediately after menopause for a short period. MHT that starts many years after menopause for a long period of time has been associated with more side effects; WHI data showed that MHT was not associated with major complications in women (50-59 years of age) 6 . 
Other studies 7-9 have generated interest in MHT due to encouraging results with a beneficial effect on coronary artery disease; Hormone therapy started immediately after menopause (up to 7 months postmenopause) significantly reduces mortality from coronary artery disease.

It also showed a lower incidence of heart failure and myocardial infarction; without increasing the risk of thromboembolic events, stroke, or cancer; in women with an average age of 50 years, within the first 3 years of menopause. The progression of the thickness of the carotid artery intimamedia and atherosclerosis was evaluated by means of the calcium score in the coronary artery; not finding an increase in the carotid intima-media thickness or a higher incidence of atherosclerosis, which supports the concept of the window of opportunity, this is when the initiation of MHT in early postmenopause has a net benefit. 9. MHT reduces risk of cardiovascular disease only if used in this "window of opportunity" period. Therefore, to reduce the risk of coronary heart disease and general mortality in women, the best strategy would be to start hormone therapy within 10 years of menopause and / or before 60 years of age, for a short period of time. MHT after 60 years of age is at risk; due to advanced age, the vascular lumen is partially obliterated with atherosclerotic plaques; MHT will make the plaque unstable, reducing vasodilation, which will eventually increase the risk of cardiovascular disease 10; the effects of using THM 11 for the primary or secondary prevention of CVD or for the preservation of cognitive function was not indicated, it should be noted that only $30 \%$ of women were 50 to 59 years of age at the beginning of the study, the incidence of Venous thromboembolism increased, while no other risk was observed. Initiating MHT early, before atherosclerosis has established itself, helps slow its progression.

\section{Endogenous Estrogen and Comorbidities}

Endogenous estrogen has many beneficial and protective effects, delaying the onset of age-related comorbidities. Due to hypoestrogenism in menopause, the risk of vasomotor symptoms (VMS), osteoporosis, cardiovascular disease, cancer and dementia increases. Estrogen prevents atherosclerosis, inflammatory changes and has a favorable effect on lipids with high and low density lipoproteins (HDL and LDL), thus avoiding cardiovascular events. Estrogen acts on the endothelial vessels and smooth muscles causing vasodilation, and preventing cardiovascular risks.

\section{Biomarkers of Vasomotor Symptoms of Cardiovascular Diseases and the Window of Opportunity}

Evidence suggests that SVMs could be considered biomarkers of cardiovascular disease 12. THM is effective in controlling SVMs associated with menopause at any age. Comorbidities can be prevented if MHT is started for symptomatic women before age 60 or within 10 years after menopause. Non-hormonal options can be used only if MHT is contraindicated or if the patient does not want it. SVMs could be a biomarker of cardiovascular diseases and will help in the selection of patients for the longest duration of THM $[5,6]$.

\section{THM on vasomotor symptoms, mood swings and osteoporosis}

The most recent evidence suggests that all women with SVM are likely to have lower bone mineral density (BMD); women aged 50-79 years with moderate / severe SVM had lower BMD in the femoral neck and lumbar spine, increased rates of hip fractures during more than 8 years of followup compared to women who did not have SVM 13. Focus on these symptomatic women for the prevention of osteoporosis-related morbidity and mortality, MHT decreases the risk of fractures. MHT can be started in postmenopausal women at risk of fracture or osteoporosis in the window of opportunity, starting after 60 years requires an individualized approach [1-6].
THM helps improve mood swings in early postmenopausal women. THM may also be helpful for women transitioning to menopause (TM) with major depression. Some women with suicidal tendencies improve with MHT, but antidepressant therapy should be the first option in this context in consultation with a psychiatrist [6].

\section{Redution of comorbidities related to the administration, routes and duration of $\mathrm{MHT}$}

There is a large body of high-quality evidence showing that estrogen-only MHT decreases the incidence of myocardial infarction and also mortality from other causes when initiated in women younger than 60 years and / or within 10 years of menopause. But estrogen-only therapy is not recommended when the uterus is intact, so the addition of progesterone is mandatory to prevent the development of endometrial cancer (EC).

Evidence on estrogen plus progestogen therapy initiated in women younger than 60 years or within 10 years of menopause shows mixed results compared to the estrogen-alone group. To reduce progesterone-related morbidity, the strategy of using lipid-friendly molecules such as natural progesterone and dydrogesterone may be preferred; However, the risk of arterial and venous thromboembolism (VTE) has not been reduced with oral THM.

The increased risk of breast cancer (MC) is associated with the use of a medroxyprogesterone. MHT started in early menopause has shown evidence that it can prevent Alzheimer's disease in later life 14. Oral MHT started in older women has shown an increased risk of dementia.

The world consensus recommends that MHT be used in low doses for the shortest possible duration before the age of 60 years or within 10 years of menopause; women who have VMS beyond the age of 60 may require THM; if it is needed beyond the age of 60 , then an individual risk calculation must be performed to keep morbidities to a minimum; A recent study changed the belief that VMS are short-lived and disappear within 2 years. VMS can last for many years; therefore, they may not be adequately included in the "short-lived symptoms" in menopause.

The VMS lasted more than 7 years; in more than $50 \%$ of women, persisting for 4.5 years after the last menstrual period [15]. In women between 65 and 79 years, the prevalence of MSV was $39.2 \%$, the highest prevalence of VMS was observed in women between 65 and 69 years 16 and it is not recommended to routinely discontinue systemic estrogen at the age of 65 years. The dose, type and route of administration of MHT should be selected according to the treatment objectives, the choice of patients and risk factors; therefore, it must be individualized [1-6].

The risk profile must be reassessed individually on an annual basis. Some women may require it for a longer time. The decision on how, when to start MHT, and when to stop it should be made after discussion with the patient, and should be done under the supervision of an expert. Hormone therapy can be harmful in women who have pre-existing coronary artery disease, cerebrovascular disease, or thromboembolic tendencies. In the presence of high risk factors if THM is indicated, transdermal preparations are preferred. Women should be aware of the non-hormonal therapies available for the management of SVM associated with TM and early menopause. Premature menopause requires a standard THM dose prescribed until the age of natural menopause and then discontinued with an informed decision.

\section{Discussion}

MHT is used to alleviate menopausal symptoms and maintain BMD [1$5,17]$, it can reduce cardiovascular risk, particularly if it is started early after menopause 18. Estrogen is required with the addition of a progestogen to women with a uterus 17. Although the inclusion of progestogens in THM regimens is associated with approximately one case of breast cancer per 
1000 women per year for current users of THM, micronized progesterone or dydrogesterone 19 may be associated with a lower risk [20,21].

The type of progestogen is also important when considering the risk of VTE, micronized progesterone and dydrogesterone which appear to show the lowest risk [22]. CEE and CEE / MPA regimens have favorable effects on lipids, but progestogens have little effect on estrogen-induced reductions in total and LDL cholesterol [23,24]. Dydrogesterone does not appear to oppose the potentially beneficial effects of estradiol on insulin [25].

There is no current guidance on the choice of progestogen for use in HRT in the UK; therefore, individual risk factors, patient preference, and clinical setting must be taken into account [26-28].

\section{Conclusion.}

THM should not be used routinely for the primary or secondary prevention of CVD; the window of opportunity or time hypothesis has cardiovascular benefits when MHT is started immediately after menopause. The role of natural progesterone versus synthetic progestins needs further evaluation. Healthy lifestyles and alternative strategies should be encouraged to reduce cardiovascular risk in menopausal women. The prescription of THM improves the quality of life. The initiation, route, and duration of MHT should be individualized for each patient after appropriate counseling and a thorough risk-benefit discussion. An early start of MHT in women selected in the window of opportunity is the key to having the best balance of risks and benefits to prevent all menopause and MHT-related comorbidities.

\section{References}

1. Avis N, Crawford S, Greendale G et al. Duration of menopausal vasomotor symptoms over the menopause transition. JAMA Intern Med 2015; 175 (4): 531-539.

2. NICE. Menopause: diagnosis and management. NICE Guideline 23. NICE, 2015.

3. NHS. Hormone replacement therapy (HRT). www.nhs.uk/conditions/hormone-replacement-therapy-hrt/ (accessed 17 July 2019).

4. Baber R, Panay N, Fenton A; IMS Writing Group. 2016 IMS recommendations on women's midlife health and menopause hormone therapy. Climacteric 2016; 19 (2): 109-150.

5. Vinogradova Y, Coupland C, Hippisley-Cox J. Use of hormone replacement therapy and risk of venous thromboembolism: nested case-control studies using the QResearch and CPRD databases. BMJ 2019; 364: k4810.

6. Suvarna Satish Khadilkar. Post-reproductive Health: Window of Opportunity for Preventing Comorbidities.The Journal of Obstetrics and Gynecology of India (January-February 2019) 69(1):1-5

7. Schierbeck LL, Rejnmark L, Tofteng CL, et al. Effect of hormone replacement therapy on cardiovascular events in recently postmenopausal women: randomised trial. BMJ. 2012;9(345):e6409.

8. Wolff EF, He Y, Black DM, et al. Self-reported menopausal symptoms, coronary artery calcification, and carotid intimamedia thickness in recently menopausal women screened for the Kronos early estrogen prevention study (KEEPS). Fertil Steril. 2013;99(5):1385-1391.

9. De Villiers TJ, Hall JE, Pinkerton JV, et al. Revised global consensus statement on menopausal hormone therapy. Climacteric. 2016;19:313-315.
10. Hodis HN, Mack WJ, Henderson VW, et al. Vascular effects of early versus late postmenopausal treatment with estradiol. N Engl J Med. 2016;374(13):1221-1231.

11. Marjoribanks J, Farquhar C, Roberts H, et al. Long-term hormone therapy for perimenopausal and postmenopausal women. Cochrane Database Syst Rev. 2017.

12. Biglia N, Cagnacci A, Gambacciani M, et al. Vasomotor symptoms in menopause: a biomarker of cardiovascular disease risk and other chronic diseases? Climacteric. 2017;20(4):306312.

13. Crandall CJ, Aragaki A, Cauley JA, et al. Associations of menopausal vasomotor symptoms with fracture incidence. J Clin Endocrinol Metab. 2015;100:524-534.

14. Wharton W, Gleason CE, Miller VM, et al. Rationale and design of the Kronos Early Estrogen Prevention Study (KEEPS) and the KEEPS cognitive and affective sub study (KEEPS Cog). Brain Res. 2013;13(1514):12-17.

15. Avis NE, Crawford SL, Greendale G, et al. Duration of menopausal vasomotor symptoms over the menopause transition. JAMA Intern Med. 2015;175:531-539.

16. Zeleke BM, Bell RJ, Billah B, et al. Vasomotor and sexual symptoms in older Australian women: a cross-sectional study. Fertil Steril. 2016;105:149-155.

17. NICE. Menopause: diagnosis and management. NICE Guideline 23. NICE, 2015.

18. Rossouw J, Prentice R, Manson J et al. Postmenopausal hormone therapy and risk of cardiovascular disease by age and years since menopause. JAMA 2007; 297 (13): 1465-1477.

19. Manson J, Chlebowski R, Stefanick M et al. Menopausal hormone therapy and health outcomes during the intervention and extended poststopping phases of the Women's Health Initiative randomized trials. JAMA 2013; 310 (13): 1353-1368.

20. Fournier A, Berrino F, Clavel-Chapelon F. Unequal risks for breast cancer associated with different hormone replacement therapies: results from the E3N cohort study. Breast Cancer Res Treat 2008; 107 (1): 103-111.

21. Lyytinen H, Pukkala E, Ylikorkala O. Breast cancer risk in postmenopausal women using estradiol- $\square$ progestogen therapy. Obstet Gynecol 2009; 113 (1): 65-73.

22. Canonico M, Oger E, Plu-Bureau G et al. Hormone therapy and venous thromboembolism among postmenopausal women. Circulation 2007; 115 (7): 840-845.

23. Lobo R, Bush T, Carr B et al. Effects of lower doses of conjugated equine estrogens and medroxyprogesterone acetate on plasma lipids and lipoproteins, coagulation factors, and carbohydrate metabolism. Fertil Steril 2001; 76 (1): 13-24.

24. Godsland I. Effects of postmenopausal hormone replacement therapy on lipid, lipoprotein, and apolipoprotein (a) concentrations: analysis of studies published from 19742000. Fertil Steril 2001; 75 (5): 898-915

25. Crook D, Godsland I, Hull J, Stevenson J. Hormone replacement therapy with dydrogesterone and 17 beta-oestradiol: effects on serum lipoproteins and glucose tolerance during 24 month follow up. Br J Obstet Gynaecol 1997; 104 (3): 298-304.

26. Avis N, Crawford S, Greendale G et al. Duration of menopausal vasomotor symptoms over the menopause transition. JAMA Intern Med 2015; 175 (4): 531-539.

27. NICE. Menopause: diagnosis and management. NICE Guideline 23. NICE, 2015.

28. NHS. Hormone replacement therapy (HRT). 


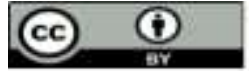

This work is licensed under Creative

Commons Attribution 4.0 License

To Submit Your Article Click Here: Submit Manuscript

DOI: $10.31579 / 2642-9756 / 039$
Ready to submit your research? Choose Auctores and benefit from:

* fast, convenient online submission

* rigorous peer review by experienced research in your field

* rapid publication on acceptance

* authors retain copyrights

* unique DOI for all articles

* immediate, unrestricted online access

At Auctores, research is always in progress.

Learn more www.auctoresonline.org/journals/women-health-care-andissues 